DOI: $10.15393 /$ j3.art.2019.4610

UDC 517.977

A. N. KiriLlov

\title{
THE METHOD OF NORMAL LOCAL STABILIZATION
}

\begin{abstract}
A problem of nonlinear systems stabilization is studied. Admissible controls are piecewise constant. The notion of normal local stabilizability is proposed. A point $P$ (not necessary equilibrium) is normally locally stabilizable if for any $\tau>0$ there exists such neighborhood $D(P ;<\tau)$ of $P$ that any point $x \in D(P ;<\tau)$ can be steered, in a time less than $\tau$, to any neighborhood of $P$ and remains there. The constructive method of normal local stabilization of nonlinear autonomous systems is presented. This method involves a special sequence of contracting cylinders containing a trajectory. A domain of attraction of a given point is constructed.
\end{abstract}

Key words: dynamical system, positive basis, normal stabilization 2010 Mathematical Subject Classification: $34 H 15$

1. Introduction. The notion of normal stabilization, introduced in this article, is motivated by the concept of normal local controllability, introduced by N. N. Petrov [6].

Definition 1. A system $\dot{x}=f(x, u), x \in \mathbb{R}^{n}$, is normally locally controllable at a point $P$ if for each $\tau>0$ there exists a neighborhood $D(P ;<\tau)$ of $P$ such that any point $x \in D(P ;<\tau)$ can be steered to $P$, in a time less than $\tau$, by an admissible control $u$.

In case of $n=2$, for analytical $f$ and piece-wise constant control N. N. Petrov obtained the necessary and sufficient conditions of the normal local controllability in terms of serial expansion coefficients for $f$ [7]. For $n>2$ such conditions are not yet found.

Definition 2. A system $\dot{x}=f(x, u), x \in \mathbb{R}^{n}$, is normally locally stabilizable at a point $P$ if for each $\tau>0$ there exists a neighborhood $D(P ;<\tau)$ of $P$ such that any point $x \in D(P ;<\tau)$ can be steered by an admissible control to any neighborhood of $P$, in a time less than $\tau$, and remains there.

(c) Petrozavodsk State University, 2019 
This notion does not deal necessarily with the equilibrium state stabilization and, therefore, it differs from the finite-time stability notion [4]. The concept of normal stabilization is useful for the control of hybrid systems, which structure may be time variant and the controller has to be able to stabilize the desired state in some constrained time. Some ideas of normal stabilization appeared in [5], though this notion was not formulated there.

The admissible control is supposed to be a piecewise constant function of time with values in $\left\{u_{i}, i=1, \ldots, n+1\right\}$, where $u_{i} \in \mathbb{R}^{m}$ are constant vectors. Given a control system $\dot{x}=f(x, u), x \in \mathbb{R}^{n}$, we prove that if the vectors $f\left(0, u_{i}\right), i=1, \ldots, n+1$, affinely generate $\mathbb{R}^{n}$, then the point $x=0$ can be normally stabilized by admissible control. The sufficient condition for normal stabilization means that $\left\{f\left(0, u_{i}\right), i=1, \ldots, n+1\right\}$ is the positive basis of $\mathbb{R}^{n}[1]$, i. e. any $x \in \mathbb{R}^{n}$ may be represented as $x=\sum_{i=1}^{n+1} \lambda_{i} f\left(0, u_{i}\right)$ with nonnegative $\lambda_{i} \in \mathbb{R}$.

The main result of the article is the constructive method of normal local stabilization. It is worth to note that the result of N. N. Petrov [6], [7] is not constructive. He proved the existence of control in the problem of normal local controllability, using his implicit positive function theorem.

The method of normal stabilization, presented in this article, may be used in problems of stabilization via sliding mode [2], [3]. But, unlike [3], where the method of stabilization is constructed under the crucial obtuse condition, i.e. the angles between vectors of a special positive basis are obtuse, in this article the angles between vectors $f\left(0, u_{i}\right), i=1, \ldots, n$, are arbitrary.

The structure of the article is organized as follows. In Section 2 we give some definitions, present properties of the positive basis and introduce some geometric objects. Section 3 is devoted to the construction of the main instrument of the normal stabilization method which is the cylinder of stabilization. The method of normal stabilization, based on the sequence of cylinders of stabilization, is presented in Section 4. Some examples are given in Section 5 .

2. Positive basis. Consider the nonlinear control system

$$
\dot{x}=f(x, u),
$$

where $x(t) \in \mathbb{R}^{n}$ is the state, $u(t) \in U \subset \mathbb{R}^{m}$ is the control input. Let $f: \mathbb{R}^{n} \times \mathbb{R}^{m} \rightarrow \mathbb{R}^{n}$ be Lipschitz with respect to $x$. Denote by $x^{\mathrm{T}} y$ the scalar product, $\|x\|^{2}=x^{\mathrm{T}} x$, where $\mathrm{T}$ is the symbol of transposition. The admissible control $u(t)$ is a piecewise constant function. 
Definition 3. The set $\left\{a_{j}\right\}, j=1, \ldots, k, a_{j} \in \mathbb{R}^{n}$, is called a positive basis of $\mathbb{R}^{n}$ if for each $x \in \mathbb{R}^{n}$ there exist such $\lambda_{j} \in \mathbb{R}, \lambda_{j} \geqslant 0, j=1, \ldots, k$, that $x=\sum_{j=1}^{k} \lambda_{j} a_{j}$.

Definition 4. The set $\left\{a_{i}\right\}, i=1, \ldots, m$, is called a positive basis of a hyperplane $\pi_{n-1} \in \mathbb{R}^{n}$ if for each $x \in \pi_{n-1}$ there exist such $\lambda_{i} \in \mathbb{R}, \lambda_{i} \geqslant 0$, $i=1, \ldots, m$, that $x=\sum_{i=1}^{m} \lambda_{i} a_{i}$.

It is easy to see that that the minimum positive basis of $\mathbb{R}^{n}$ consists of $(n+1)$ vectors and for $\pi_{n-1}$ it consists of $n$ vectors.

Lemma 1. The set $\left\{a_{1}, \ldots, a_{n+1}\right\}$ is the positive basis of $\mathbb{R}^{n}$ if and only if the following both statements are true.

1. The origin $O \in \mathbb{R}^{n}$ is the interior point of the convex hull of $a_{1}, \ldots, a_{n+1}: O \in \operatorname{int}\left(\operatorname{co}\left(a_{1}, \ldots, a_{n+1}\right)\right)$.

2. Any $n$ vectors among $a_{1}, \ldots, a_{n+1}$ are linear independent.

Lemma 2. If $\left\{a_{1}, \ldots, a_{n+1}\right\}$ is the positive basis of $\mathbb{R}^{n}$ then any $a_{i}, i=$ $=1, \ldots, n+1$ cannot be expressed as the nonnegative linear combination of the other vectors of the positive basis.

The proofs of Lemmas 1, 2 simply follow from the definition [1].

Let $a_{i}, i=1, \ldots, n$, be linear independent. Denote by $\mathrm{A}=\left(a_{1}, \ldots, a_{n}\right)$ the matrix with the coordinate columns $a_{1}, \ldots, a_{n}$ in some basis. We put $\alpha=\left(\alpha_{1}, \ldots, \alpha_{n}\right)^{\mathrm{T}} \geqslant 0(>0)$, if $\alpha_{i} \geqslant 0(>0), i=1, \ldots, n$. Denote by $K$ and $K^{*}$ the open cone and the conjugate open cone, respectively, spanned by $a_{1}, \ldots, a_{n}$

$$
K=\left\{x \in \mathbb{R}^{n}: x=\mathrm{A} \alpha, \alpha>0\right\}, \quad K^{*}=\left\{y \in \mathbb{R}^{n}: \mathrm{A}^{\mathrm{T}} y<0\right\},
$$

and let $\bar{K}, \bar{K}^{*}$ be the closures of $K$ and $K^{*}$, respectively

$$
\bar{K}=\{x: x=\mathrm{A} \alpha, \alpha \geqslant 0\}, \quad \bar{K}^{*}=\left\{y: \mathrm{A}^{\mathrm{T}} y \leqslant 0\right\} .
$$

Denote $\widetilde{K}=\mathbb{R}^{n} \backslash(\bar{K} \cup(-\bar{K}))$.

Lemma 3. Assume that $a_{1}, \ldots, a_{n}, a_{i} \in \mathbb{R}^{n}, n \geqslant 2$, are linear independent and $-K^{*} \subset K$ with $K, K^{*}$ defined in (2). Then for any $y \in K^{*}$ there exists a hyperplane $\pi_{n-1}(y) \subset \widetilde{K}$ such that $z^{\mathrm{T}} y=0$ for each $z \in \pi_{n-1}(y)$.

Proof. Since $-y \in-K^{*} \subset K$ then there exists $\alpha>0$ such that $-y=\mathrm{A} \alpha$ and $\mathrm{A}^{\mathrm{T}}(-y)>0$. Hence $\mathrm{A}^{\mathrm{T}} \mathrm{A} \alpha>0$. The linear independence of $a_{1}, \ldots, a_{n}$ implies the existence of such $\gamma \in \mathbb{R}^{n}$ that $z=\mathrm{A} \gamma$. Then we obtain

$$
z^{\mathrm{T}}(-y)=(\mathrm{A} \gamma)^{\mathrm{T}} \mathrm{A} \alpha=\gamma^{\mathrm{T}} \mathrm{A}^{\mathrm{T}} \mathrm{A} \alpha=\gamma_{1} \beta_{1}+\ldots+\gamma_{n} \beta_{n}
$$


where $\beta_{i}, \gamma_{i}$ are the components of vectors $\mathrm{A}^{\mathrm{T}} \mathrm{A} \alpha, \gamma$, respectively, $\beta_{i}>0$. In order to satisfy $z^{\mathrm{T}} y=0$ we can put, for example, $\gamma_{l}=-\gamma_{s} \beta_{l} / \beta_{s}, \gamma_{i}=0$, $s \neq i \neq l, \gamma_{s} \neq 0$ for any fixed $l, s \in\{1, \ldots, n\}$.

Remark. If $-K \subset K^{*}$ we assume $\widetilde{K}=\mathbb{R}^{n} \backslash\left(\bar{K}^{*} \cup\left(-\bar{K}^{*}\right)\right)$, and Lemma 3 is true in this case for any $y \in K$.

Definition 5. Vector $y$ is called a normal to plane $\pi_{n-1}(y)$.

Lemma 4. Assume that $a_{1}, \ldots, a_{n}$ are linear independent and $-K^{*} \subset K$. Let $b_{i}=b_{i}(y)$ be the orthogonal vector projection of $a_{i}$ onto $\pi_{n-1}(y)$ with $y \in K^{*}$. Then $\left\{b_{1}, \ldots, b_{n}\right\}$ is the positive basis of $\pi_{n-1}(y)$.

Proof. First, we prove that the origin $O$ is the interior point of the convex hull of $b_{i}, i=1, \ldots, n$. Let $l(O)$ be the straight line with directing vector $y \in K^{*}$, containing $O$. Denote by $e_{i}$ the orthogonal projection of $a_{i}$ onto $l(O): e_{i}=\left(a_{i}^{\mathrm{T}}(-e)\right)(-e)=-k_{i} y$, where $e=y /\|y\|, \quad k_{i}=$ $=\left(a_{i}^{\mathrm{T}}(-e)\right) /\|y\|>0$. Then $a_{i}=b_{i}+e_{i}$ and

$$
a_{i}=b_{i}-k_{i} y, i=1, \ldots, n \text {. }
$$

Multiplying equalities (3) by $\alpha_{i}>0$, adding them and taking into account that $-y=\alpha_{1} a_{1}+\ldots+\alpha_{n} a_{n}$ with $\alpha_{i}>0$, we obtain $(\widetilde{k}-1) y=\alpha_{1} b^{1}+$ $+\ldots+\alpha_{n} b^{n}$, where $\widetilde{k}=\alpha_{1} k_{1}+\ldots+\alpha_{n} k_{n}$. Multiplying the latter equality by $y^{T}$, we obtain $(\widetilde{k}-1) y^{\mathrm{T}} y=0$. Since $y \neq 0$ then $\widetilde{k}=1$ and therefore $\alpha_{1} b^{1}+\ldots+\alpha_{n} b^{n}=0$, where $\alpha_{i}>0$, which means that $O$ is the interior point of the convex hull of $b_{i}, i=1, \ldots, n$.

Secondly, let us prove that any $n-1$ vectors among $b_{1}, \ldots, b_{n}$ are linearly independent. Assume, without loss of generality, that $b_{1}, \ldots, b_{n-1}$ are linearly dependent. Then there exist $\delta_{j}, j=1, \ldots, n-1$, such that $\delta_{1} b_{1}+\ldots+\delta_{n-1} b_{n-1}=0$ for $\delta_{j} \in \mathbb{R}, \delta_{1}^{2}+\ldots+\delta_{n-1}^{2} \neq 0$. Using (3) we obtain that $\left(\delta_{1}-k \alpha_{1}\right) a_{1}+\ldots+\left(\delta_{n-1}-k \alpha_{n-1}\right) a_{n-1}-k \alpha_{n} a_{n}=0$, where $k=\delta_{1} k_{1}+\ldots+\delta_{n-1} k_{n-1}$. Since $a_{i}, i=1, . ., n$, are linearly independent and $\alpha_{i}>0$, we obtain that $k=\delta_{1}=\ldots=\delta_{1}=0$. Therefore $b_{1}, \ldots, b_{n}$ are linearly independent. The conclusion of lemma follows from Lemma 1 . $\square$

Definition 6. Let us call $b_{1}, \ldots, b_{n}$ a projective positive basis of $\pi_{n-1}(y)$ corresponding to a basis $a_{1}, \ldots, a_{n}$.

Remark. The similar result remains valid in the case $-K \subset K^{*}, y \in K$.

Denote $a_{i}(M)=f\left(M, u_{i}\right)$, where $u_{i} \subset U$ are any constant vectors, $i=1, \ldots, n+1$, and denote by $K(M ; j)$ a cone with vertex $M$, spanned 
by vectors $a_{i}(M), i=1, \ldots, n+1, i \neq j$,

$$
K(M ; j)=\left\{x \in \mathbb{R}^{n}: x=\sum_{i=1, i \neq j}^{n+1} \alpha_{i} a_{i}(M), \alpha_{i}>0\right\} .
$$

Assume that there exists a neighborhood $D(O)$, a ball of center $O$, such that for any $M \in D(O):\left\{a_{1}(M), \ldots, a_{n+1}(M)\right\}$ is the positive basis of $\mathbb{R}^{n}$.

Lemma 5. If $M \in D(O)$ then

1. $K(M ; j) \bigcap K(M ; s)=\emptyset$ if $j \neq s, j, s=1, \ldots, n+1$;

2. $\bigcup_{j=1}^{n+1} \bar{K}(M ; j)=\mathbb{R}^{n}$;

3. $\bigcap_{j=1}^{n+1} \bar{K}(M ; j)=M$.

The proof of this lemma, with slight modification, see in [3].

3. Cylinder of Stabilization. In what follows, without loss of generality, we consider the normal stabilization of the origin. The following assumptions are hold valid throughout the paper

$$
M \in D(O), \quad O \in \bar{K}(M ; j),
$$

where $D(O), \bar{K}(M ; j)$ are described above. Next, assume that

$$
\left\|a_{i}(M)\right\| \geqslant \Delta, i=1, \ldots, n+1
$$

for some $\Delta>0$.

Let $K^{*}(M ; j)$ be a conjugate open cone with respect to $K(M ; j)(2)$. Consider the case $-K^{*}(M ; j) \subset K(M ; j)$ for some $j \in I=\{1, \ldots, n+1\}$. Then for any $y(M) \in K^{*}(M ; j)$

$$
a_{i}^{\mathrm{T}}(M) y(M)<0, i=1, \ldots, n+1, i \neq j .
$$

In what follows, $y(M)$ is a unit vector: $\|y(M)\|=1$. The finiteness of the set $\left\{a_{i}(M), i=1, \ldots, n+1\right\}$ implies the existence of constant $d(M)>0$ such that

$$
a_{i}^{\mathrm{T}}(M) y(M) \leqslant-d(M), i=1, \ldots, n+1, i \neq j .
$$

Assume that there exist $y \in K^{*}(M ; j)$ which does not depend on $M$ (but not on $j$ ) and $d>0$ which does not depend on $M$ and $j$ such that for each $M \in D(O)$

$$
a_{i}^{\mathrm{T}}(M) y \leqslant-d, i=1, \ldots, n+1, i \neq j .
$$


For instance, the continuity of $a_{i}(M)$ implies that if $\left\{a_{i}(O), i=1, \ldots, n+1\right\}$ is the positive basis then such $y$ and $d>0$ exist. Naturally, such $y$ and $d$ are not unique. Denote by $l(M ; y)$ the straight line through $M$ with directing vector $y$, and let $\pi_{n-1}(M ; y)$ be a plane through $M$ with normal vector $y$, i. e. $\pi_{n-1}(M ; y)$ is a plane orthogonal to $l(M ; y)$. For $i \neq j$ let $e_{i}(M)$ be a vector projection of $a_{i}(M)$ on $l(M ; y)$. Then

$$
a_{i}(M)=b_{i}(y)+e_{i}(M),
$$

where $\left\{b_{i}(y), i=1, \ldots, n+1, i \neq j\right\}$ is a projective positive basis of $\pi_{n-1}(M ; y)$ corresponding to a basis $\left\{a_{i}(M), i=1, \ldots, n+1, i \neq j\right\}$.

Lemma 6. If $y$ satisfies (6) then

$$
\min _{i \in I, i \neq j}\left\|e_{i}(M)\right\| \geqslant d
$$

Proof. Since

$$
e_{i}(M)=\left(a_{i}^{\mathrm{T}}(M)(-y)\right)(-y)
$$

then $\left\|e_{i}(M)\right\|=\left|a_{i}^{\mathrm{T}}(M) y\right| \geqslant d$.

Let us construct the stabilization cylinder $C$. Denote by $\pi_{n-k}(M ; y)$ a plane through $M$ of codimension $k, k \in\{1, \ldots, n-1\}$, with normal vector $y$. Denote $N=l(M ; y) \cap \pi_{n-1}(O ; y)$. In what follows we need $N \neq O$. If $N=O$ we can take another $y \in K^{*}(M ; j)$, which is not unique.

Introduce the balls

$$
\begin{aligned}
& D_{n}(O ; N)=\left\{x \in \mathbb{R}^{n}:\|x\| \leqslant\|O N\|\right\} \\
& D_{n-1}(O ; N)=D_{n}(O ; N) \cap \pi_{n-1}(O ; y) .
\end{aligned}
$$

Let $[M N]$ be the segment with $M$ and $N$ as the endpoints.

Definition 7. Let us call the set

$$
C=D_{n-1}(O ; N) \times[M N] \subset D(O)
$$

the cylinder of stabilization, with the bottom base $D_{n-1}^{b}=D_{n-1}(O ; N)$ and the top base $D_{n-1}^{t}=C \bigcap \pi_{n-1}(M ; y)$.

Remark. The cylinder of stabilization $C$ is not unique, because $y$ is not unique. Moreover, we assume that $D(O)$ is so large that $C \subset D(O)$.

Denote by $S_{n-2}^{t}, S_{n-2}^{b}$ the spheres which are the boundaries of the balls $D_{n-1}^{t}, D_{n-1}^{b}$, respectively. Let $\pi_{n-2}(M)$ be the $(n-2)$-dimensional 
plane through $M$, tangent to $S_{n-2}^{t}$. Obviously, $\pi_{n-2}(M) \subset \pi_{n-1}(M ; y)$. Let $x\left(t, t_{0}, M, u\right)$ be the trajectory of the system (1), corresponding to a control $u$, such that $x\left(t_{0}, t_{0}, M, u\right)=M$.

Lemma 7. There exists such constant control $u=u_{s} \in U, s \in I$, that the trajectory $x\left(t, t_{0}, M, u_{s}\right)$ of the system (1) enters $C$ at the moment $t=t_{0}$, i.e. $M$ is the ingress point.

Proof. Let $\left\{b_{i}(y)\right\}$ be the projective basis corresponding to $a_{i}(M), i \neq$ $j, i=1, \ldots, n+1$, i.e. the positive basis of $\pi_{n-1}(M ; y)$. Hence the vectors $b_{i}(y)$ cannot be directed from $M$ into one half plane of $\pi_{n-1}(M ; y)$ with the boundary $\pi_{n-2}(M)$ [1], then there exists $b_{s}(y)$ for some $s \in I$, which is transversal to $S_{n-2}^{t}$ and is directed into the interior of $D_{n-1}^{t}$. The corresponding $u_{s}$ is the required control. Really, let $\pi_{n-1}([M N])$ be the hyperplane tangent to $C$ such that $[M N] \subset \pi_{n-1}([M N])$. Denote by $p(M)$ a vector orthogonal to $\pi_{n-1}([M N])$ and such that $b_{s}(y)^{T} p(M)<0$. Multiplying scalarly the equality $a_{s}(M)=b_{s}(y)+e_{s}(M)$ by $p(M)$ we obtain that $a_{s}(M)^{\mathrm{T}} p(M)<0$. Taking in account that $a_{s}^{\mathrm{T}}(M) y \leqslant-d$, we obtain that the trajectory $x\left(t, t_{0}, M, u_{s}\right)$ enters the cylinder $C$ when $t=t_{0}$, i. e. at point $M$.

Lemma 8. There exists the admissible (piecewise constant) control $u \in U$ such that the trajectory $x\left(t, t_{0}, M, u\right)$, remaining in $C$, reaches the bottom base $D_{n-1}^{b}$ of $C$ in a finite time.

Proof. Let us note that the trajectory $x\left(t, t_{0}, M, u_{s}\right)$, where $u_{s}$ is the control from Lemma 7 , intersects any hyperplane parallel to $\pi_{n-1}(O ; y)$ in the direction of the half-space containing the origin $O$. It follows from Lemma 6. If $x\left(t, t_{0}, M, u_{s}\right)$ reaches $D_{n-1}^{b}$, remaining in $C$, then this lemma is proved. Suppose that $M_{1}$ is the next point (after $M$ ) at which the trajectory $x\left(t, t_{0}, M, u_{s}\right)$ hits the boundary of $C$ before attaining $D_{n-1}^{b}$. Then, similarly to the proof of Lemma 7 , it can be proved that there exists a constant control $u_{s 1} \in U$ such that the trajectory $x\left(t, t_{0}, M_{1}, u_{s}\right)$ enters $C$ at a point $M_{1}$. Let us switch the control from $u_{s}$ to $u_{s 1}$ at the moment when the trajectory hits the boundary of $C$ at $M_{1}$. Use this control till the trajectory hits boundary of $C$ at some point $M_{2}$ before attaining $D_{n-1}^{b}$. Then switch the control from $u_{s 1}$ to $u_{s 2}$ with which the trajectory enters $C$ at a point $M_{2}$ and so on. We obtain the control sequence $u_{s}, u_{s 1}, u_{s 2}, \ldots$. Lemma 6 implies that there exists $u_{s l} \in U$ with which the trajectory reaches $D_{n-1}^{b}$ in a finite time. 
Remark. In the case $-K(M ; j) \subset K^{*}(M ; j)$ the proof is analogous to the above one.

\section{Method of normal stabilization.}

Definition 8. A point $M$ is $\tau$-reducible if there exists an admissible control $u \in U$ such that $M$ can be steered by $u$, along a trajectory of the system (1), to any neighborhood of $O$, in a time less than $\tau$, and remains there.

Definition 9. The domain of $\tau$-reducibility of the origin is the set of all $\tau$-reducible points.

Theorem 1. Assume that conditions (4), (5), (6) are fulfilled. If

$$
\|O M\|<\tau d(1-\delta)
$$

where $\delta \in(0,1)$ is a constant determined in the proof, then $M$ is a $\tau$-reducible point.

Proof. Let us construct the cylinder $C$ as it was described in the previous section. Introduce the radius $r_{0}=\|O N\|$ of the base and the height $h_{0}=\|N M\|$ of the cylinder $C$. As it was proved (Lemmas 7,8 ) there exists a piecewise constant $u \in U$ such that the trajectory $x\left(t, t_{0}, M, u\right)$ reaches the base $D_{n-1}^{b}$ of $C$ at some point $M_{1} \in D_{n-1}^{b}$ in finite time $\tau_{0}$ : $x\left(t_{0}+\tau_{0}, t_{0}, M, u\right)=M_{1}$. Then, considering $M_{1}$ as the initial point, we can construct the cylinder $C_{1}$, the construction of which is analogous to $C$. In addition, suppose that $O \in K\left(M_{1} ; j_{1}\right), j_{1} \in\{1, \ldots, n+1\}$. The trajectory $x\left(t, t_{0}+\tau_{0}, M_{1}, u\right)$ reaches the low base of $C_{1}$ in some finite time $\tau_{1}$. Introduce the radius $r_{1}=\left\|O N_{1}\right\|$ of the base and the height $h_{1}=\left\|N_{1} M_{1}\right\|$ of the cylinder $C_{1}$, where $N_{1}$ is the point belonging to the bottom base of $C_{1}$ and the vector $N_{1} M_{1}$ is orthogonal to the base of $C_{1}$. Proceeding this procedure we obtain the sequences $\tau_{k}, r_{k}=\left\|O N_{k}\right\|$, $h_{k}=\left\|N_{k} M_{k}\right\|, O \in K\left(M_{k} ; j_{k}\right), j_{k} \in I, k=0,1,2, \ldots, M_{0}=M, N_{0}=N$, $j_{0}=j$. Let us prove that $M_{k} \rightarrow O$, when $k \rightarrow \infty$. Since

$$
O M_{k}=N_{k} M_{k}+O N_{k}
$$

where $N_{k} M_{k}$ is a vector orthogonal to $O N_{k}$, then $\left\|O M_{k+1}\right\| \leqslant\left\|O N_{k}\right\|$. Thus, denoting $d_{k}=\left\|O M_{k}\right\|=\sqrt{h_{k}^{2}+r_{k}^{2}}$, we obtain

$$
d_{k+1} \leqslant r_{k}<d_{k}
$$


Multiplying (9) scaralry by $O N_{k}$, we obtain

$$
\left(O M_{k}^{\mathrm{T}}\right)\left(O N_{k}\right)=\left\|O N_{k}\right\|^{2}>0 .
$$

Hence

$$
\left\|O N_{k}\right\|=\frac{\left(O M_{k}^{\mathrm{T}}\right)\left(O N_{k}\right)}{\left\|O N_{k}\right\|}=\frac{\left(O M_{k}^{\mathrm{T}}\right)\left(O N_{k}\right)\left\|O M_{k}\right\|}{\left\|O N_{k}\right\| \cdot\left\|O M_{k}\right\|} .
$$

Thus $r_{k}=\delta_{k} d_{k}$, where $\delta_{k}=\left(\left(O M_{k}^{\mathrm{T}}\right)\left(O N_{k}\right)\right) /\left(\left\|O N_{k}\right\| \cdot\left\|O M_{k}\right\|\right)>0$. Vectors $O M_{k}, O N_{k}$ are not collinear. Therefore the Cauchy inequality implies that $\delta_{k}<1$.

Since $O \in K\left(M_{k}, j_{k}\right)$ then there exist such $\alpha_{i}^{k} \geqslant 0, i=1, \ldots, n+1$, that

$$
M_{k} O=\sum_{i=1}^{n+1} \alpha_{i}^{k} a_{i}\left(M_{k}\right), \quad i \neq j_{k} \in I .
$$

Assume, on the contrary, that $M_{k} \nrightarrow O$ as $k \rightarrow \infty$. Condition (5) and nonnegativeness of $\alpha_{i}^{k}$ imply that there exists $i_{k} \neq j_{k}$ such that $\alpha_{i_{k}}^{k} \geqslant \eta$ for some $\eta>0, k=0,1,2, \ldots$. Multiplying (9) scaralry by $\left(-y_{k}\right)$ where $y_{k}$ is a unit normal vector corresponding to a cylinder $C_{k}$ (like $y$ corresponds to $C$ ), taking in account that $a_{i}\left(M_{k}\right)\left(-y_{k}\right) \geqslant d$ (Lemma 6 and assumption (6)), we obtain

$$
M_{k} O\left(-y_{k}\right)=\sum_{i=1}^{n+1} \alpha_{i}^{k} a_{i}\left(M_{k}\right)\left(-y_{k}\right) \geqslant d \sum_{i=1}^{n+1} \alpha_{i}^{k} \geqslant d \eta,
$$

or

$$
d_{k} \cos \beta_{k} \geqslant d \eta
$$

where $\cos \beta_{k}=M_{k} O\left(-y_{k}\right) /\left\|M_{k} O\right\|$ is a cosine of an acute angle $\beta_{k}$ between $M_{k} O$ and $-y_{k}$. Since the sequence $\left\{d_{k}\right\}$ is bounded then the latter inequality implies the existence of such $\bar{\beta}$ that $0<\beta_{k} \leqslant \bar{\beta}<\frac{\pi}{2}$. Then

$$
\cos \left(\frac{\pi}{2}-\beta_{k}\right) \leqslant \cos \left(\frac{\pi}{2}-\bar{\beta}\right)=\sin \bar{\beta} .
$$

Denote $\delta=\sin \bar{\beta}<1$. Hence $\delta_{k} \leqslant \delta<1$ for any $k=1,2, \ldots$, and $r_{k} \leqslant \delta d_{k}$. Then (10) implies that $d_{k+1} \leqslant \delta d_{k}$ and therefore $d_{k+1} \leqslant \delta^{k+1} d_{0}$. Thus $d_{k} \rightarrow 0$ as $k \rightarrow \infty$, and $M_{k} \rightarrow O$, which contradicts to assumption.

Now let us prove that for any $\tau>0$ there exists $U(O ;<\tau)$ such that for any point $M_{0} \in U(O ;<\tau): \sum_{1}^{\infty} \tau_{k}<\tau$. Since $\tau_{k}$ is the time of moving from $M_{k-1}$ to $M_{k}$, then, according to Lemma 6 ,

$$
\tau_{k} \leqslant h_{k-1} / d<d_{k-1} / d \leqslant\left(d_{0} \delta^{k-1}\right) / d .
$$


Therefore,

$$
\sum_{k=1}^{\infty} \tau_{k} \leqslant\left(d_{0} / d\right) \sum_{k=1}^{\infty} \delta^{k-1}=d_{0} /(d(1-\delta)) .
$$

Hence if $d_{0}=\left\|O M_{0}\right\|<\tau d(1-\delta)$, then $\sum_{k=1}^{\infty} \tau_{k}<\tau$. Thus, $M_{0}=M$ is a $\tau$-reducible point. Thus the point $O$ is normally stabilized if

$$
M_{0} \in U(O ;<\tau)=U_{r}(O) \cap U(O ; d)
$$

for $r<\tau d(1-\delta)$, where $U_{r}(O)$ is a ball of radius $r$ centered at the origin.

If $M_{k}=O$ for some $k$ then we move a little bit from $M_{k}$ to some point $\widetilde{M}_{k}$ and then use the procedure described above.

Remark 1. The procedure described above implies $C_{k} \subset U_{\left\|M_{k} O\right\|}(O)$.

Remark 2. The origin may be replaced by any point $M$ with a positive basis $\left\{f\left(M, u_{i}\right), i=1, \ldots, n+1\right\}$.

5. Examples. In this section we present three examples to illustrate the results discussed above.

Example 1. Consider the system

$$
\begin{gathered}
\dot{x}_{1}=g_{1}\left(x_{1}, x_{2}\right)+u-u^{3}, \quad \dot{x}_{2}=g_{2}\left(x_{1}, x_{2}\right)+1-u^{2}, \\
u \in\{-2,0,2\},
\end{gathered}
$$

where $x_{1}, x_{2} \in \mathbb{R}, g_{1}(0,0)=g_{2}(0,0)=0, g_{1}, g_{2}$ are the Lipschitz functions. Consider $f\left(x_{1}, x_{2}, u\right)=\left(f_{1}, f_{2}\right)$, where $f_{1}, f_{2}$ are the right-hand sides of equations (12). The vectors

$$
f(0,0,0)=(0,1), \quad f(0,0,2)=(-6,-3), \quad f(0,0,-2)=(6,-3)
$$

form the positive basis of $\mathbb{R}^{2}$. Therefore, the origin $O$ is normally locally stabilizable.

Example 2. Consider the Euler equations for an angular velocity of a rigid body

$$
\begin{gathered}
I_{1} \dot{\omega}_{1}=\left(I_{2}-I_{3}\right) \omega_{2} \omega_{3}+u_{1}, \quad I_{2} \dot{\omega}_{2}=\left(I_{3}-I_{1}\right) \omega_{1} \omega_{3}+u_{2}, \\
I_{3} \dot{\omega}_{3}=\left(I_{1}-I_{2}\right) \omega_{1} \omega_{2}+u_{3},
\end{gathered}
$$

where $\omega_{i}=\omega_{i}(t)$ are the components of the angle velocity vector in a fixed coordinate system coinciding with the principal axes, $I_{i}$ are the principal inertia moments, $i=1,2,3$. The applied torque $u=\left(u_{1}, u_{2}, u_{3}\right)$ is 
a piecewise constant control. If the admissible constant controls $u^{j}=$ $=\left(u_{1 j}, u_{2 j}, u_{3 j}\right), j=1,2,3,4$, form the positive basis of $\mathbb{R}^{3}$ then the origin $O$ is normally locally stabilizable. For example,

$$
u^{1}=(1,0,0), \quad u^{2}=(0,1,0), \quad u^{3}=(0,0,1), \quad u^{4}=(-1,-1,-1) .
$$

Example 3. Consider the system

$$
\dot{x}_{1}=x_{1}+\cos u, \quad \dot{x}_{2}=x_{1} x_{2}+\sin u, \quad u \in\{\pi / 3 ; 2 \pi / 3 ; 3 \pi / 2\} .
$$

Denote $x=\left(x_{1}, x_{2}\right), O=(0,0), f_{1}(x)=f(x, \pi / 3), f_{2}(x)=f(x, 2 \pi / 3)$, $f_{3}(x)=f(x, 3 \pi / 2)$. The vectors $f_{i}(O)$ form the positive basis of $\mathbb{R}^{2}$. Therefore, the origin $O$ is normally locally stabilizable.

Let us construct the cylinder $C$. At first, we find the open set $\Pi$, $O \in \Pi \subset \mathbb{R}^{2}$, such that for any $x \in \Pi$ the vectors $f_{i}(x), i=1,2,3$, form the positive basis. The conditions of collinearity for pairs of the vectors $f_{i}(x), f_{j}(x), i \neq j$ are as follows:

1. $f_{1}(x), f_{2}(x)$ are collinear if and only if $x_{1} x_{2}=-\frac{\sqrt{3}}{2}$;

2. $f_{1}(x), f_{3}(x)$ are collinear if and only if $x_{2}=\frac{1}{x_{1}}+2+\sqrt{3}$;

3. $f_{2}(x), f_{3}(x)$ are collinear if and only if $x_{2}=\frac{1}{x_{1}}-(2+\sqrt{3})$.

Therefore, the continuity of $f_{i}(x)$ implies that

$$
\Pi=\left\{\left(x_{1}, x_{2}\right): x_{1} x_{2}>-\frac{\sqrt{3}}{2}, x_{2}<\frac{1}{x_{1}}-(2+\sqrt{3}), x_{2}>\frac{1}{x_{1}}+2+\sqrt{3}\right\} .
$$

Let us take, for example, the initial point $M_{0}=(0.1 ; 0.1) \in \Pi$ and construct $C$ for it. Since $O \in K\left(M_{0} ; 1\right)$, we consider the hyperplane $\pi(O ; y)$ with normal vector $y=(a, b)$, satisfying $y^{\mathrm{T}} f_{i}(O)<0, i=2,3$. Therefore, $0<b<\frac{a}{\sqrt{3}}$. Now, take, for example, $(a, b)=(1,0.25), d=0.125$ and find the set $\widetilde{\Pi}(d) \supset U(O ; d)$, for which $y^{\mathrm{T}} f_{i}(x)<0, i=2,3$ :

$$
x_{1}-\frac{1}{2}+\frac{1}{4}\left(x_{1} x_{2}+\frac{\sqrt{3}}{2}\right)<-\frac{1}{8}, \quad x_{1}+\frac{1}{4}\left(x_{1} x_{2}-1\right)<-\frac{1}{8},
$$

which leads to $\widetilde{\Pi}(d)=\left\{x: 8 x_{1}+2 x_{1} x_{2}<1\right\}$. Then we obtain the hyperplanes, containing the bases of $C$, e. g. $\pi\left(M_{0} ; y\right): x_{2}+4 x_{1}=0$, $\pi(O ; y): x_{2}+4 x_{1}=0.5$. Now we can construct the cylinder

$$
C=\left\{x: x_{2} \geqslant-4 x_{1}, x_{2} \leqslant-4 x_{1}+1, x_{2} \geqslant \frac{1}{4} x_{1}-\frac{1}{8}, x_{2} \leqslant \frac{1}{4} x_{1}+\frac{3}{40}\right\} .
$$


Instead of $C$, constructed above, we can take any cylinder (rectangular), containing in $C$, with basis on straight lines $x_{2}+4 x_{1}=0, x_{2}+4 x_{1}=0.5$ and the lateral sides parallel to $x_{2}=0.25 x_{1}-0.125, x_{2}=0.25 x_{1}+0.075$.

6. Conclusions. In this article the notion of normal stabilization of control systems is proposed. An admissible control is piece-wise constant. The constructive method of normal stabilization, based on a sequence of cylinders of stabilization, is presented. An extension of the presented approach to non-autonomous systems is the subject of future investigations.

Acknowledgment. This work was supported by RFBR (18-01-00249a).

\section{References}

[1] Davis C. Theory of positive linear dependence. Amer. J. Math., 1954, vol. 76, pp. 733-746.

[2] Utkin V. I. Sliding Modes in Control and Optimization. Springer-Verlag, 1992.

[3] Bartolini G., Pisano A., Punta E., Usai E. A survey of applications of second order sliding mode control to mechanical systems. Int. J. Control, 2003, vol 76, pp. 875-892.

[4] Bhat S. P., Bernstein D. S. Finite-time stability of autonomous systems. SIAM J. Control Optim., vol. 38, pp. 751-766.

[5] Kirillov A. N. The stabilization problem for certain class of ecological systems. Int. J. Softw. Eng. Knowl. Eng., 1997, no. 7, pp. 247-251.

[6] Petrov N. N. Local controllability of autonomous systems. Differ. Uravn. (in Russian), 1968, no. 7, pp. 1218-1232.

[7] Petrov N. N. A solution of a certain problem in control theory. Differ. Uravn. (in Russian), 1969, no. 5, pp. 962-963.

Received April 1, 2018.

In revised form, July 10, 2018.

Accepted August 14, 2018.

Published online January 5, 2019.

Institute of Applied Mathematical Research

of the Karelian Research Centre of the Russian Academy of Sciences

11, Pushkinskaya str., Petrozavodsk 185910, Russia;

Petrozavodsk State University

33 Lenina pr., Petrozavodsk 185910, Russia

E-mail: krllv1812@yandex.ru 